\title{
Non-adiabatic rotational excitation of dipolar molecule under the influence of delayed pulses
}

\author{
URVASHI ARYA $^{\mathrm{a}}$, BRIJENDER DAHIYA ${ }^{\mathrm{b}}$ and VINOD PRASAD ${ }^{\mathrm{b}, *}$ \\ ${ }^{a}$ Department of Physics and Astrophysics, University of Delhi, Delhi 110 007, India \\ ${ }^{b}$ Department of Physics, Swami Shraddhanand College, University of Delhi, Delhi 110 036, India \\ e-mail: urvashivarsheney@gmail.com; brijender.dahiya@gmail.com; vprasad@ss.du.ac.in; \\ vijit_vin@yahoo.co.in
}

MS received 16 April 2012; revised 2 January 2013; accepted 28 February 2013

\begin{abstract}
We suggest a control scheme for choosing populations of molecular rotational states by wave packet interference. The rotational wave packets of $\mathrm{LiCl}$ molecule excited non-adiabatically by half cycle pulse (HCP) is controlled using the second ultrashort HCP. By adjusting the time delay between the two laser pulses, constructive or destructive interference among these wave packets enables the population to be enhanced or repressed for the specific rotational state. The role played by the field strength and the pulse duration is also calculated numerically. We have used fourth order Runge-Kutta method to study non-adiabatic rotational excitation (NAREX) dynamics.
\end{abstract}

Keywords. NAREX; delay time; wave packet interference.

\section{Introduction}

The dynamics of molecule in the external electromagnetic field has recently been a subject of many experimental and theoretical study. ${ }^{1-11}$ Interaction between the field and molecule can be studied either under adiabatic or non-adiabatic conditions. When the interaction is shorter than the duration of its rotational period, then non-adiabatic rotational excitation (NAREX) occurs. ${ }^{12-18}$ According to rough estimate for NAREX, pulse duration is $\left(T_{\text {pulse }}<h / B\right)$, where $h$ is Planck's constant and $B$ is rotational constant of the molecule. The time evolution is said to be non-adiabatic and molecule is supposed to end up in a rotational wave packet. The wave packet comprises of a finite number of free rotor states and thus recurs after the pulse has passed giving rise to orientation under field-free condition. ${ }^{19-27}$ The rotation of molecule is coherently excited to create a rotational quantum wave packet, a non-stationary super position of rotational eigen states. NAREX is useful for studies on quantum state resolved collision or reaction dynamics of highly anisotropic state. NAREX can become an important tool for changing and controlling the rotational state distribution of molecules, especially when recent elaborate methods i.e. double-pulse pair ${ }^{28}$ and shaped pulses ${ }^{29,30}$ are invoked. NAREX shows useful results in internal

*For correspondence version, ${ }^{31,32}$ dissociative ionization ${ }^{33,34}$ molecular rearrangement, ${ }^{35}$ fragmentation to neutral products, ${ }^{36}$ non-resonant electronic excitation ${ }^{37}$ etc. In addition to atom, molecule has nuclear degree of freedom (rotational and vibration) and can undergo internal conversion or dissociation of molecules, the variety of competing outcomes of NAREX must be greatly increased. NAREX has been utilized in many other applications. ${ }^{38,39}$ The non-stationary quantum state i.e. rotational wave packet, created in non-adiabatic molecular orientation is a coherent superposition of eigenstates, of which rotational angular momentum $J$ ranges among various values while its projection onto the space fixed axis $M$ is preserved to the initial value. In this respect, the non-adiabatic orientation is inherently accomplished by NAREX. An efficient method to achieve an enhanced degree of orientation is to employ delayed laser pulses. As the orientation is resulted from the in phase overlapping of spherical harmonics in the time evolution of rotational wave packets, introducing the second laser pulse may modify the rotational wave packet and thereby increases the degree of orientation. As a model scenario of coherent control of quantum states the rotational wave packet excited by the first laser pulse can be annihilated or enhanced by the second laser pulse when introduced at different delay times. The delayed pulse used here is ultrashort half cycle pulse (UHCP). These pulses are already feasible. ${ }^{40,41}$ Half cycle pulses (HCPs) with the peak 
field of up to several hundreds of $\mathrm{kV} / \mathrm{cm}$ and duration in the femtosecond regimes can be experimentally generated. ${ }^{42-46}$ Ultrashort pulses create non-stationary superposition, or wave packet, in the multilevel medium. This wave packet oscillates with a characteristic time that corresponds to the quantum beat period between the excited states. An ultra-short HCP, which is short compared to rotational periods, could impart a kick on the molecule and transfer angular momentum. The consequence would be angular motion in the direction of kick. ${ }^{47,48}$ In this paper we investigate the theoretical study on control of rotational wave packet using HCP and delayed ultrashort HCP. The rotational wave packet was calculated by using two laser pulses with different laser parameters like laser pulse duration and by applying second laser pulse at different delay times. The time evolution of the rotational wave packet was characterized by time dependent orientation parameter.

\section{Theory}

The laser induced rotational excitation dynamics is studied for $\mathrm{LiCl}$ molecule, in simultaneous presence of $\mathrm{HCP}$ and ultrashort HCP. The molecule is treated within rigid-rotor approximation (frozen internal vibrational motion), interacting with the fields. The molecule plus the two fields Hamiltonian, for this model is

$$
H(t)=B J^{2}+V_{E}(\theta, t)+V_{Z}\left(\theta^{\prime}, t\right),
$$

where, $B$, is the rotational constant and $J^{2}$ is the squared angular momentum operator. The interaction potentials $V_{E}(\theta, t)$ and $V_{Z}\left(\theta^{\prime}, t\right)$ are the interaction potential of the laser field (HCP) and ultrashort $\mathrm{HCP}$ with dipole moment and along the polarizability, respectively, where $V_{E}$, define as

$$
V_{E}(\theta, t)=\mu_{0} E(t) \cos (\theta),
$$

where $\theta$, being the polar angle between the molecular axis and the laser field. It is precisely this angle which defines the orientation of the molecule with respect to laser field and $\mu_{0}$ is the permanent electric dipole moment along the internuclear axis. $\theta$ and $\theta^{\prime}$ are in plane of each other. The laser field is defined as

$$
E(t)=E_{0} f(t) \sin (\omega t),
$$

where $E_{0}$, is the electric field amplitude; and $f(t)$ is the envelop defined as

$$
\begin{gathered}
f(t)=\sin ^{2}\left(\pi t / t_{p}\right) \quad 0<t<t_{p} \\
f(t)=0 \quad \text { otherwise. }
\end{gathered}
$$

In the equation (3), $\omega$, is the frequency of the applied field of the HCP, which fits the main features of the experimental shape peak amplitude $E_{0}$ duration $t_{P}$ (full width at half maximum, FWHM) of HCP except the tail which is dropped. The short unipolar pulse is followed by a very long, weak tail of opposite electric field with a marked asymmetry between the maximum and the minimum at either side. This tail typically lasts tens of pico seconds and has an amplitude of $10 \%$ or less than the main peak (an amplitude asymmetry of approximately 12:1). Strictly, the electric field for a freely propagating electromagnetic pulse must integrate to zero, be it over a very long time (in the case of the $\mathrm{HCP}$, the area of the tail, integrated up to $t=4 \mathrm{ps,}$ represents $15 \%$ of the area of the main peak). However, a transfer of angular momentum should take place on the time scale of the short pulse where the electric field is essentially unidirectional, and the effect of the long weak tail should be negligible as mentioned by Dion. ${ }^{49}$ Concerning the HCP field, following points may be taken care of (i) maximum field amplitude we have considered is $200 \mathrm{kV} / \mathrm{cm}$. This is quite low compared to the intensities required for the ionization of the diatomic molecule, considered here, (ii) central frequencies are out of resonance in this case. Even though there is probability flow to few of the low lying rotational states, which get modified considerably by the ultrashort HCP pulse, (iii) we have considered the pulse duration of $1.0 \mathrm{ps}$, which is quite small compared to rotational period $\left(t_{c}\right)$ of the $\mathrm{LiCl}$ molecule $(\mathrm{h} / 2 \mathrm{~B}=$ $23.6 \mathrm{ps}$ ), hence non-adiabatic interaction are observed. It has ionization energy $9.57 \mathrm{eV}$. While $V_{Z}\left(\theta^{\prime}, t\right)$, is the interaction due to ultrashort $\mathrm{HCP}$ pulse

$$
V_{z}\left(\theta^{\prime}, t\right)=\mu_{0} E_{1}(t) \cos \left(\theta^{\prime}\right),
$$

where $E_{1}(t)=\sin ^{2}\left(\pi t / T_{p}\right) ; 0<t<T_{p}, T_{p}$ is pulse duration of ultrashort HCP. $\theta^{\prime}$, is polar angle between the molecular axis and ultrashort HCP. In order to ensure that the observed rotational excitation is induced by NAREX process, a quantum dynamical calculation is performed to calculate the population by solving the time dependent Schrödinger equation (TDSE) in the presence of $\mathrm{HCP}^{50}$

$$
\iota \hbar \frac{\left|\partial \psi_{r o t}(t)\right\rangle}{\partial t}=\left(H_{r o t}-\mu \cos \theta E(t)\right)\left|\psi_{r o t}(t)\right\rangle .
$$

The time dependent rotational wave packet $\left|\psi_{\text {rot }}(t)\right\rangle$ is expanded in terms of the rotational eigenstates $|J M\rangle$ of $H_{\text {rot }}$ as

$$
\left|\psi_{r o t}^{J_{i} M_{i}}(t)\right\rangle=\sum_{J=0}^{J_{\max }} \sum_{M=-J}^{J} C_{J M}^{J_{i} M_{i}}(t)|J M\rangle e^{-i E^{J} t / h} .
$$

With the initial condition

$$
\left.\left|\psi_{r o t}^{J_{i} M_{i}}(t=0)=\right| J_{i} M_{i}\right\rangle,
$$


inserting the equations (8) and (9) into equation (7), one obtains a set of coupled equations for the timedependent expansion coefficients,

$$
\begin{aligned}
& i \hbar \frac{d C_{J M}^{J_{i} M_{i}}(t)}{d t}=-\mu E(t) \sum_{J^{\prime}=0}^{J_{\max }^{\prime}} \sum_{M^{\prime}=-J^{\prime}}^{J^{\prime}} \\
& \times\left\langle J M|\cos (\theta)| J^{\prime} M^{\prime}\right\rangle \\
& \times e^{-i\left(E^{J^{\prime}}-E^{J}\right) t / \hbar} C_{J^{\prime} M^{\prime}}^{J_{i} M_{i}}(t) \\
& C_{J M}^{J_{i} M_{i}}(t=0)=\delta_{J J_{i}} \delta_{M M_{i}} .
\end{aligned}
$$

The selection rules $J^{\prime}=J \pm 1$ and $M^{\prime}=M$ simplify the differential equation (10) as

$$
\begin{aligned}
i \hbar \frac{d C_{J M_{i}}^{J_{i} M_{i}}(t)}{d t}= & -\mu E(t) \sum_{J^{\prime}=0}^{J_{\max }^{\prime}}\left\langle J M_{i}|\cos (\theta)| J^{\prime} M_{i}\right\rangle \\
& \times e^{-i\left(E^{J^{\prime}}-E^{J}\right)^{t / \hbar}} C_{J^{\prime} M^{\prime}}^{J_{i} M_{i}}
\end{aligned}
$$

and

$$
C_{J^{\prime} M^{\prime}}^{J_{i} M_{i}}(t)=0 \text { for } M \neq M_{i} .
$$

The time-dependent rotational wave packet (8) is thus

$$
\left|\psi_{r o t}^{J_{i} M_{i}}(t)\right\rangle=\sum_{J=0}^{J_{\max }} C_{J M_{i}}^{J_{i} M_{i}}(t)\left|J M_{i}\right\rangle e^{-i E^{J} t / h} .
$$

The matrix elements $\left\langle J M_{i}|\cos (\theta)| J^{\prime} M_{i}\right\rangle$ for $J^{\prime}=J \pm$ 1 are given explicitly as

$$
\begin{aligned}
\left\langle J M_{i}|\cos (\theta)| J^{\prime} M_{i}\right\rangle= & (-1)^{M_{i}} \sqrt{(2 J+1)\left(2 J^{\prime}+1\right)} \\
& \times\left(\begin{array}{ccc}
J & 1 & J^{\prime} \\
M_{i} & 0 & -M_{i}
\end{array}\right) \\
& \times\left(\begin{array}{ccc}
J & 1 & J^{\prime} \\
0 & 0 & 0
\end{array}\right) .
\end{aligned}
$$

Equation (12) is solved by means of the Runge-Kutta method of fourth order. We have taken step size $t=4$ a.u and total propagation period is 100 ps for calculation. If another ultrashort HCP is irradiated onto the molecule at $t=t_{k}$, wave packet created by the interaction with the second pulse is expanded and solved by using the method used by Abiague and Berakdar. ${ }^{51}$ As described, the wave packet between successive pulses evolve under field free condition i.e. when single initial pulse is applied equation (7), follows initial condition defined by equation (9), however, the moment second pulse is applied the initial condition changes and follows equation (8). We have taken states up to $J=20$, i.e. total of 21 lowest rotational states as the field intensities considered here, only lowest few rotational states are populated. Although the convergent results are obtained by taking states up to $J=12$. To compute the degree of molecular orienta- tion, we calculate the time-dependent expectation value of $\langle\cos \theta\rangle$ as

$$
\langle\cos \theta(t)\rangle_{J_{i} M_{i}}=\left\langle\psi_{r o t}^{J_{i} M_{i}}(t)|\cos \theta| \psi_{r o t}^{J_{i} M_{i}}(t)\right\rangle .
$$

The corresponding thermally averaged time-dependent expectation value is given as a Boltzmann average over the initial state-selected values of equation (16)

$$
\begin{aligned}
& \langle\cos \theta(t)\rangle_{T} \\
& \quad=(1 / Z) \sum_{J=0}^{J_{\max }} P(J) \sum_{M_{J=-J}}^{J}\langle\cos \theta(t)\rangle_{M_{J}} .
\end{aligned}
$$

Here, $P(J)=\exp \left[\left(-B J(J+1) / k_{B} T\right)\right]$, is the Boltzmann distribution function associated with the rotational states, $Z=\sum_{J=0}^{J_{\max }}(2 J+1) P(J)$, is the partition function, $T$ is the temperature, $k_{B}$ is the Boltzmann constant, and $B=\hbar^{2} / 2 \mathrm{I}$ is the rotational constant of the molecule. The orientation parameter $\langle\cos \theta\rangle$ varies within the interval $[-1,1]$ and the perfect orientation is signified by the extremum value of $\langle\cos \theta\rangle$.

\section{Results and discussion}

Here we have considered the rotational excitation and orientation of $\mathrm{LiCl}$ molecule in the presence of collective consequence of HCP and a delayed pulse. Both pulses are linearly polarized and are in plane of each other. The delayed pulse used here is ultrashort HCP of peak intensity $7 \times 10^{13} \mathrm{~W} / \mathrm{cm}^{2}$ and frequency $3 \mathrm{~cm}^{-1}$. The duration of delayed pulse is $(0.2 \mathrm{ps})$, which is much smaller in comparison of initial pulse duration. The pulse width variation of initial HCP having frequency $11 \mathrm{~cm}^{-1}$ allows the system to be exposed to the electromagnetic (e.m.) field for different durations and the delayed pulse is used for the kick mechanism. Wave packet evolves as in field-free condition after second pulse, hence we have shown the results for well after the end of second pulse. This is first study regarding NAREX and orientation in the presence of pulses of different shapes and different durations. So far studies were carried out by two identical pulses ${ }^{52-54}$ but we are considering two different pulses and second pulse is ultrashort (fs) with respect to the first pulse and is ultrastrong. The consideration of $\mathrm{LiCl}$ molecule as sample is obvious, as it has been extensively studied and is an ideal case of orientation as it has large value of permanent dipole moment and polarizability. Orientation and NAREX is presented for different pulse duration $\left(t_{p}\right)$ of initial HCP and delayed pulse is applied at different delay time in comparison of rotational period $\left(t_{c}\right)$. The peak electric field strength is kept below the ionization threshold $200 \mathrm{kV} / \mathrm{cm}$, in order to study 
non-resonant laser field orientation. The results calculated in this paper are beneficial to experimental point and very useful for controlling the molecular dynamics.

In figure 1, calculations were carried out for various pulse duration of initial pulse with peak intensity of the delayed ultrashort HCP pulse fixed at $7 \times$ $10^{13} \mathrm{~W} / \mathrm{cm}^{2}$ and is applied at $t_{c} / 2$. Results shown in the figure reveals the existence of an optimal $J$ values for pulse duration. The pulse duration is indicated in each panel as: for panel (a) pulse duration is $0.1 \mathrm{ps,}$ for (b) it is $0.2 \mathrm{ps}$, for panel (c) it is $0.5 \mathrm{ps}$ and for (d) the pulse duration is $1.0 \mathrm{ps}$. Furthermore, we observe that the maximum rotational excitation value depends critically on the pulse duration. As the initial pulse duration increases; the time of exposure of the system also increases; due to this rotational probability also increases of states $J=1,2,3 \ldots$ in order, but as soon as the delayed pulse ultrashort HCP is applied, this system get kicked and all the population of rotational states get reshuffled, so that high $J$ states get excited and low $J$ states get suppressed. The rotational probability of
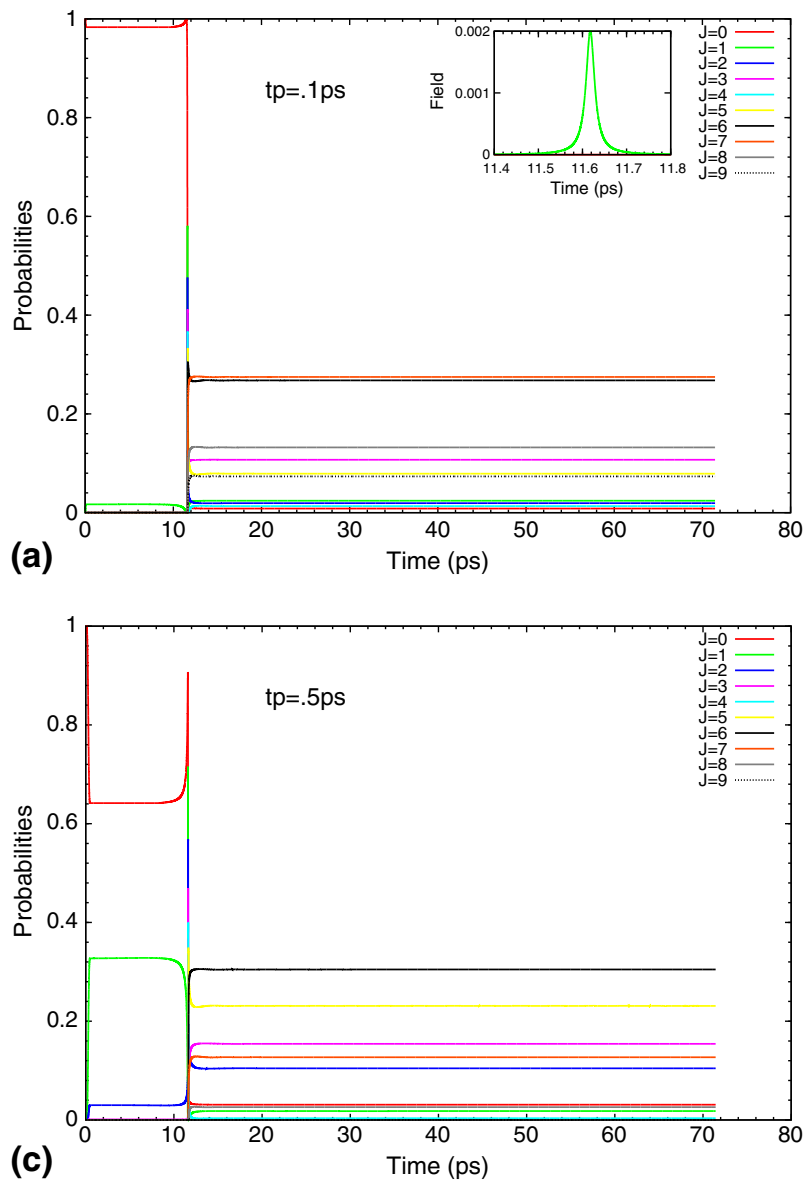

any particular state can be controlled by the change in delay time for particular delayed pulse.

In figure 2 , the transition probability of rotational state from $J=0-9$, with or without delayed pulse at different pulse duration of initial pulse indicated in each panel is shown. The $\mathrm{LiCl}$ molecule is initially in the rotational state $J=0$, when only HCP is applied. Almost all the population more than $98 \%$ is for the rotational state $J=0$, while the population of the other states is negligibly small, but as soon as the delayed ultrashort HCP introduced, the population is transferred to other higher rotational states. In panel (a), the pulse duration of initial HCP pulse is $0.1 \mathrm{ps}$, shows the maximum population transfer for the rotational state $J=7$ after applying delayed pulse. Whereas in panel (d), for initial pulse duration $1.0 \mathrm{ps}$, the maximum population transfer is for rotational state $J=4$. It is displayed in the figure that for state $J=4$ rotational population is goes on increasing with increase of pulse duration while the population of states $J=7,8,9$ goes on decreasing with the increase in the pulse duration.
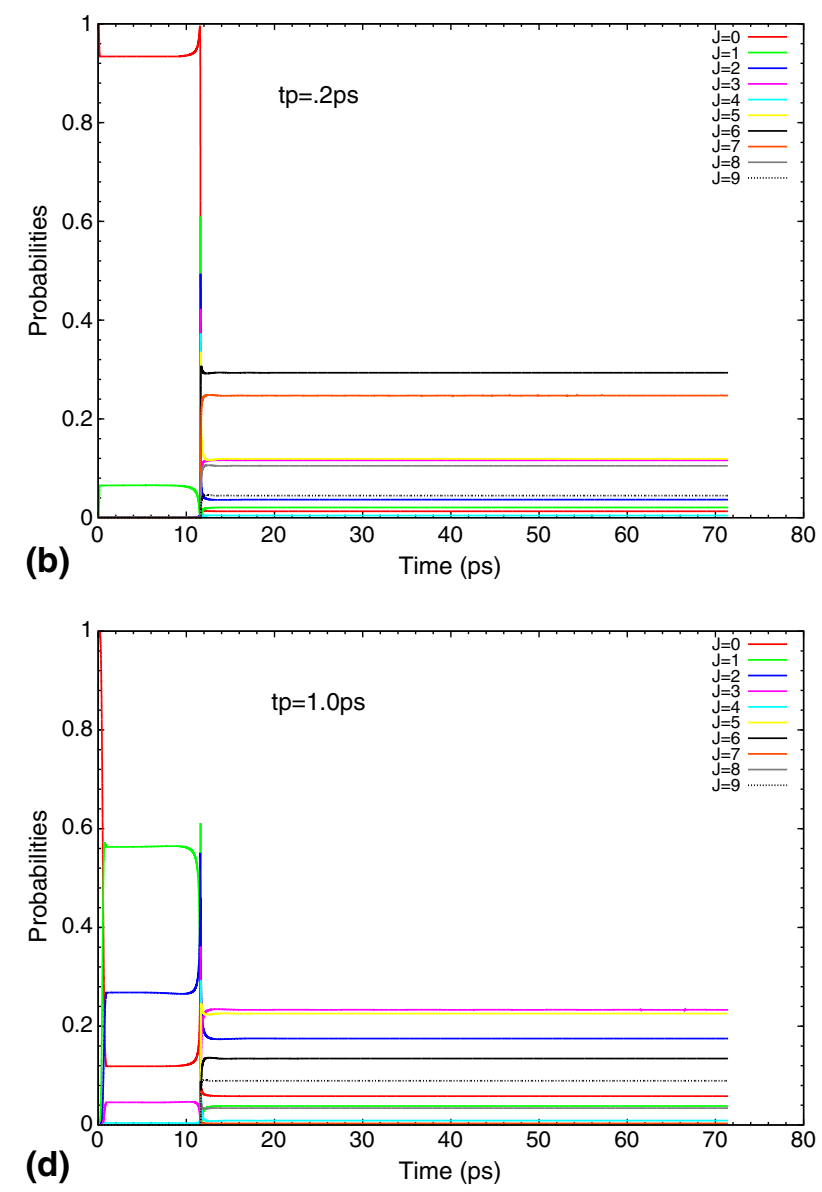

Figure 1. The time dependent rotational population with delayed ultrashort HCP applied at $t_{c} / 2$, here initial half cycle pulse duration varies from $0.1 \mathrm{ps}, 0.2 \mathrm{ps}, 0.5 \mathrm{ps}, 1.0 \mathrm{ps}$ in panel (a), (b), (c), (d), respectively. 


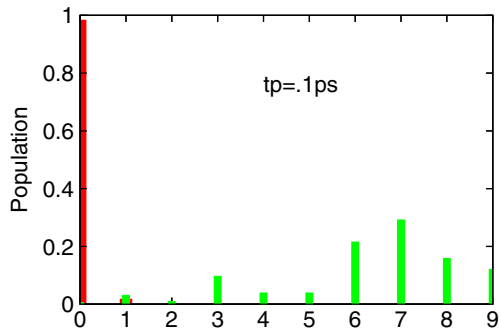

(a)

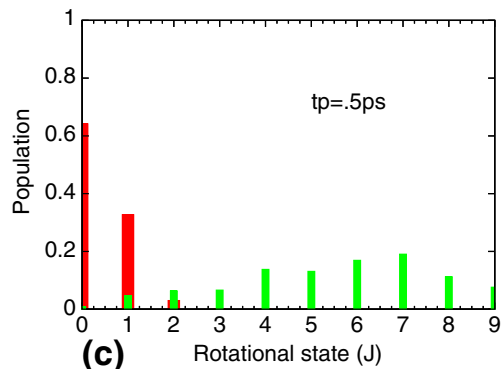

(c)

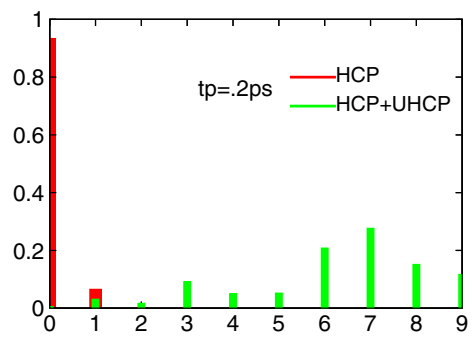

(b)

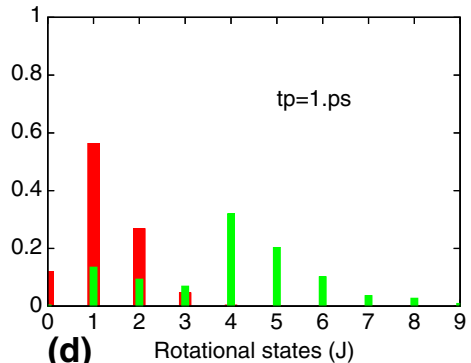

(d) Rotational states (J)

Figure 2. Transition probability of the rotational state $J=0-9$, without and with delayed ultrashort HCP at different initial pulse duration indicated in each panel. The pulse intensity of initial pulse is $200 \mathrm{kV} / \mathrm{cm}$.

In figure 3, the rotational states without external field are bare states whereas the rotational states in the presence of the external field states get dressed. Thus in this figure, we have shown bare and dressed states of different rotational states of $\mathrm{LiCl}$ molecule and the particular rotational state is indicated in each panel. Bare states
(B) are represented by red colour. Dressed states are due to two types of field, dressed states D1 shown by green colour is only due to initial HCP, while dressed states D2 shown by blue colour are the combination of both initial pulse and delayed pulse applied at $t_{c} / 2$. It is clearly shown in the figure that how the bare states get
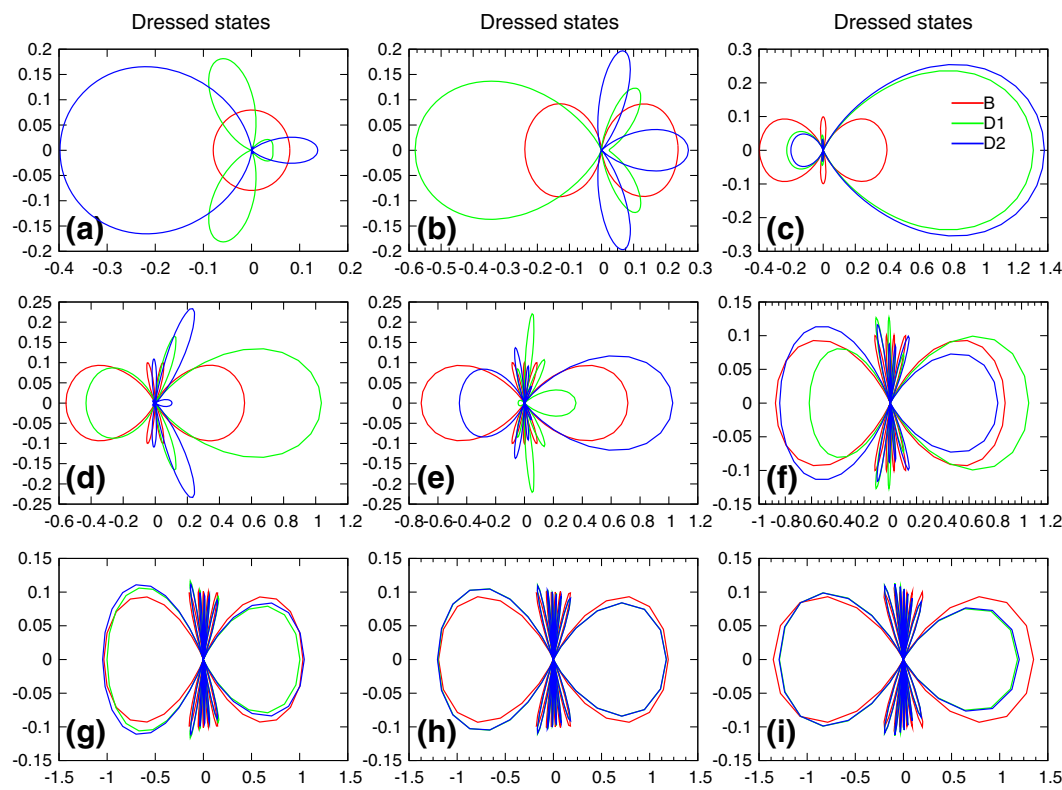

Figure 3. Bare states and dressed rotational state of molecule $J=0$ 8 , with and without delayed pulse at pulse duration $1 \mathrm{ps}$. Here, red colour represents the bare state, green colour represents dressed state with HCP only whereas blue colour represents the dressed state with both HCP and ultrashort HCP pulse. The pulse duration of initial HCP is of $1.0 \mathrm{ps}$. 

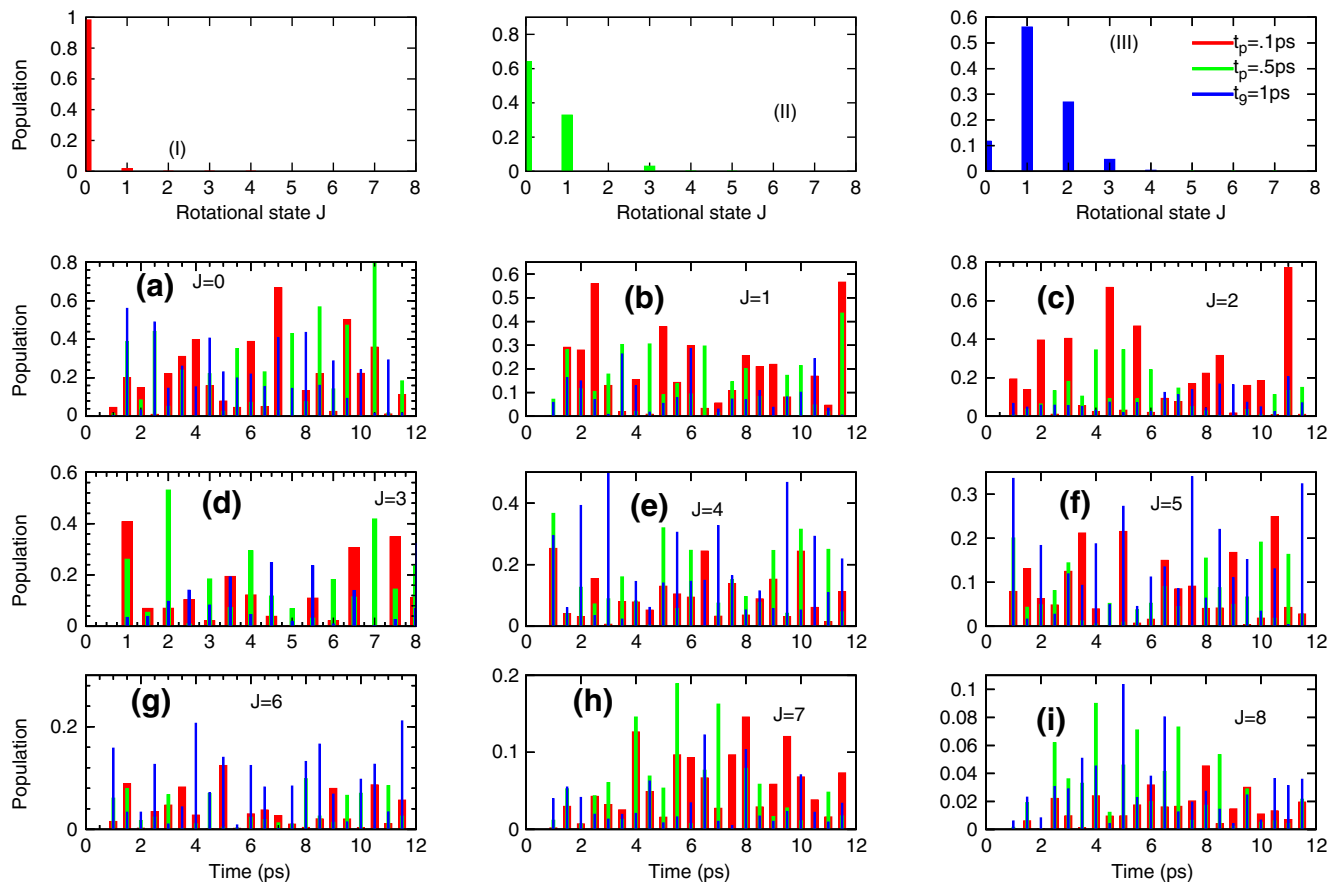

Figure 4. First row shows the rotational population with only HCP of different pulse duration indicated in the key. The rest three rows show rotational population of different $J$ states after applying delayed pulse. The three bars are for different pulse duration of initial HCP as indicated in the key of first row.

modified with external field. Mixing between the states depends upon the strength of the field, duration of HCP and the delay between the two pulses.

In figure 4; first row of the figure shows the rotational population of different $J$ states for only initial pulse of different pulse duration, as indicated in the key. Next three rows show the rotational population of a particular rotational state $J$ as a function of delay time. The three different bars are of $0.1 \mathrm{ps}, 0.5 \mathrm{ps}$ and $1.0 \mathrm{ps}$ pulse duration of initial pulse indicated in panel (iii) of first row. Here it is clear that the particular state can be controlled by the delay between the two pulses. Suppression and enhancement of the population depends on the combination of pulse duration of initial pulse and different delay times between the two laser pulses.

Figure 5 shows the population for each specific rotational state wave packet as a function of pulse duration of initial pulse only. The population is distributed among the rotational state $J=0$ to $J=5$ and rest states are almost zero. It is also found that for $t_{p}=$ $0.1 \mathrm{ps}$, most of the population is in state $J=0$, but as we increase the pulse duration the population transfers to higher rotational states. Rotational population of any particular ' $J$ ' state shows resonance for a particular
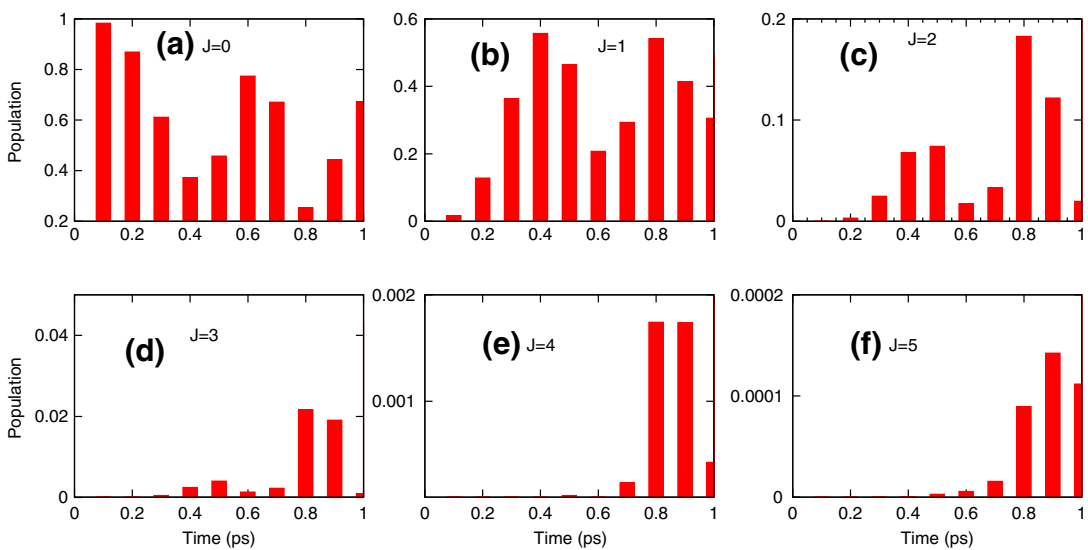

Figure 5. Population of rotational states with pulse duration of HCP pulse without delayed pulse. Rest states have almost zero values, hence not shown. 

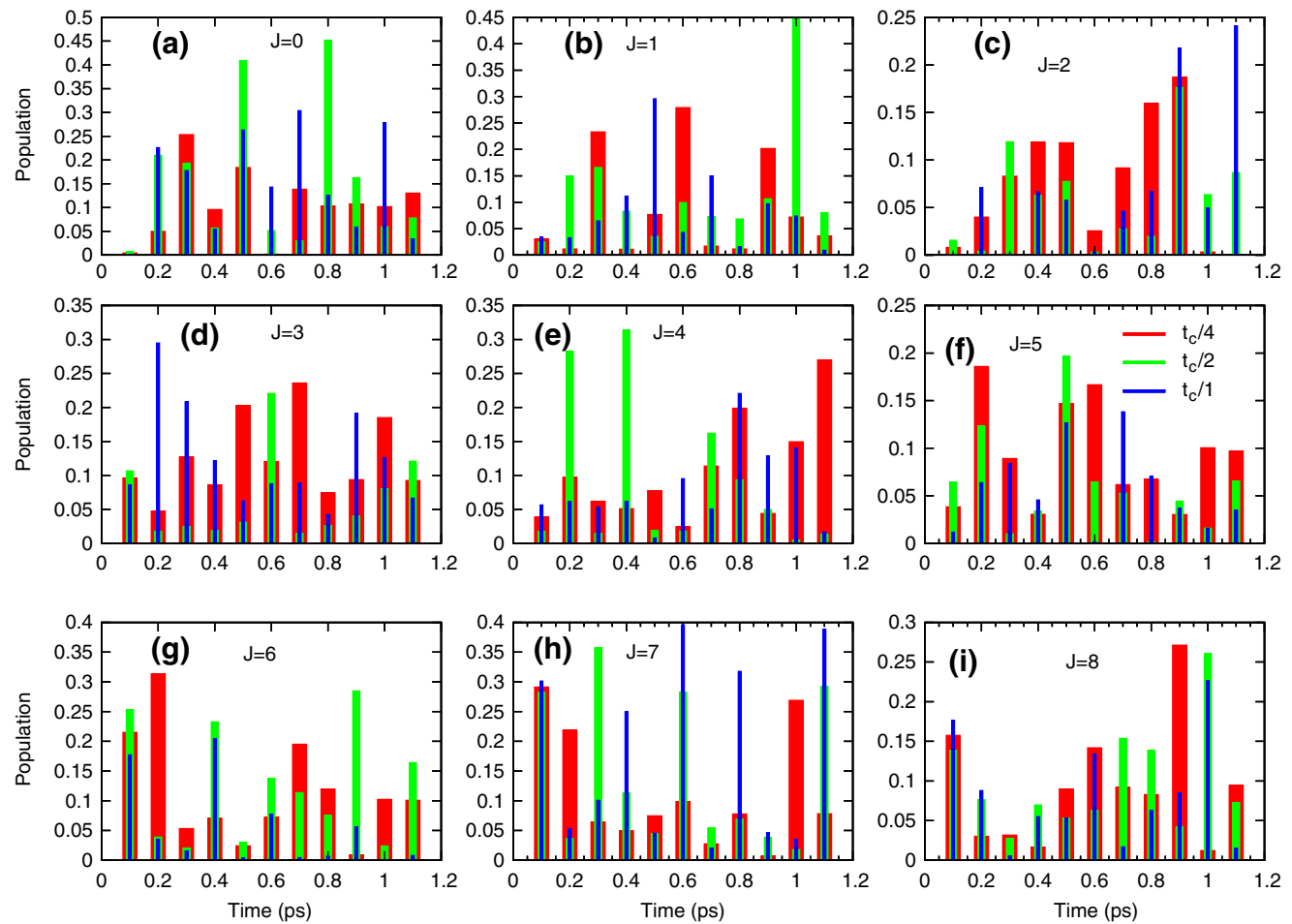

Figure 6. Population of rotational states with pulse duration of initial HCP pulse after applying delayed pulse at different delay of ultrashort $\mathrm{HCP}$ applied at $t_{c} / 4, t_{c} / 2$ and $t_{c}$ as per key.

time period; such as $J=0$, state shows resonance for $t_{p}=0.1 \mathrm{ps}$ and $0.6 \mathrm{ps}$, whereas $J=1$ state shows resonance for $t_{p}=0.4 \mathrm{ps}$ and $0.8 \mathrm{ps}$. States $J=2,3$ and 4 show maximum population for $t_{p}=0.8$ ps. So, we can say that the rotational population of higher $J$ states can only be achieved for greater exposure time and with some particular values of $t_{p}$ satisfying its resonance condition.

In figure 6, the variation of rotational population with pulse duration of initial pulse and for different delay time of delayed ultrashort $\mathrm{HCP}$ is shown. By applying the delayed ultrashort HCP pulse we get the rotational population to even $J=8$, which was not possible with only HCP as shown in figure 5. The three bars are for different delay time as indicated in the key in panel (f). The rotational population of the state can be controlled by the delay as well as pulse duration of the initial pulse. Such as for $J=0$ state; if the pulse duration $\left(t_{p}\right)$ of the initial $\mathrm{HCP}$ is 0.5 ps then the maximum rotational population is for delay time $t_{c} / 2$, but for $t_{p}=1 \mathrm{ps}$ we
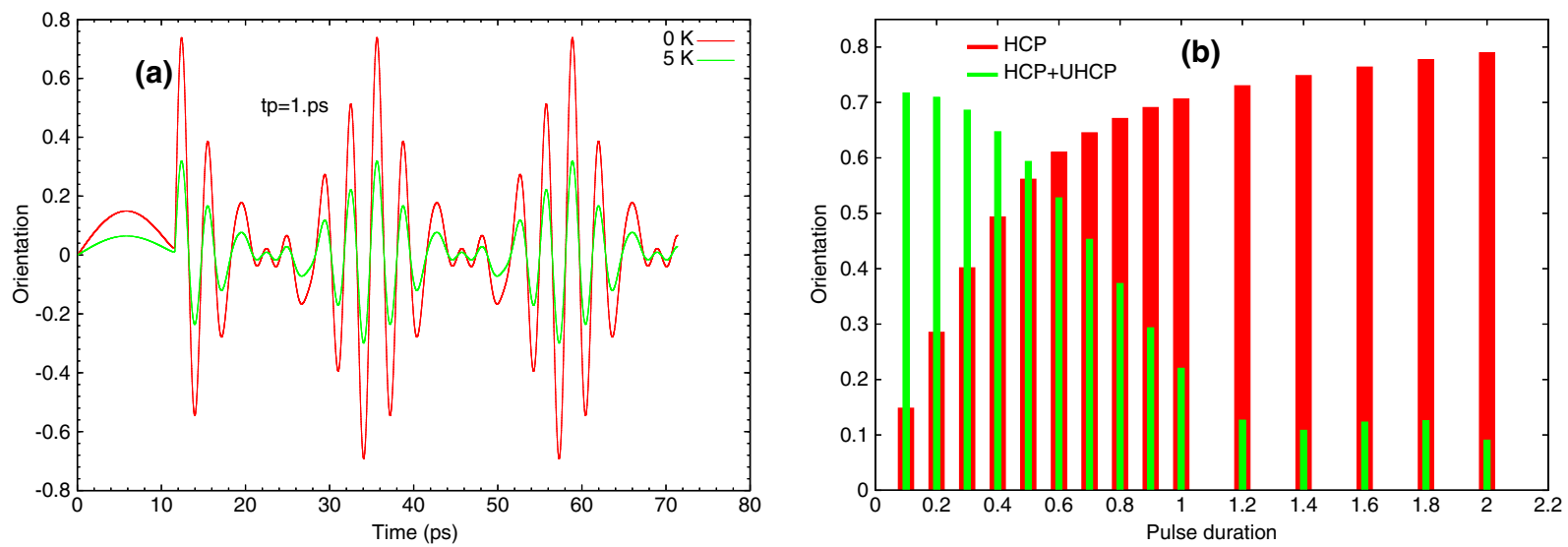

Figure 7. (a) Time dependent orientation parameter $\langle\cos \theta\rangle$ calculated at $T=0 \mathrm{~K}$ (in red) and $T=5 \mathrm{~K}$ (in green). (b) Maximum value of orientation, without delayed pulse (in red) and with delayed pulse (in green) is applied at $t_{c} / 2$. 
need a delay time $t_{c}$. Similarly for other state, we can get a combination of $t_{p}$ and delay time to attain a maxima.

Figure $7 \mathrm{a}$ shows the time evolution of orientation at two different rotational temperatures $(T=0 \mathrm{~K}$ and $T=5 \mathrm{~K}) . T=0 \mathrm{~K}$ corresponds to the case, in which all the initial rotational states were populated at the ground state. But in the real experiments multiple rotational states are populated incoherently, when molecular ensemble is at finite temperature. Eventhough value of orientation decreases with increase in temperature. Maximum value of orientation does not depend upon the initial rotational temperature. But it is nearly fixed at the half of the rotational period of the molecule.

In figure $7 \mathrm{~b}$, the maximum value of orientation with pulse duration is shown. Maximum value of orientation increases with increase in the pulse duration of HCP. But when the delayed ultrashort HCP is applied at delay of $t_{c} / 2$, the phenomenon gets almost reversed; it is observed that there is continuous decrease in maximum value of orientation with the pulse duration. It is concluded that with delayed ultrashort HCP maximum value of orientation is obtained for small exposure time of initial pulse.

\section{Conclusion}

We have calculated the rotational population of molecule selected by the quantum interference of the rotational wave packets that are created by NAREX of the molecule using two laser pulses (half cycle pulse and ultrashort HCP pulse). It is shown that rotational population can be enhanced or suppressed for specific rotational state in the wave packet created by two laser pulses at different pulse duration and at different delay times. Dressed rotational states of the molecule are evaluated with and without delayed ultra short pulse. The maximum value of molecular orientation is achieved at different pulse durations.

The delayed pulse can transfer the population selectively in the direction either further to the rotational states excited by the first pulse or back to its initial state, depending on the delay between the two pulses, resulting in the enhancement or suppression of the molecular orientation. Hence, the double pulse excitation method supports to simultaneously control of the internal quantum state distribution of reactant. This scheme can be generally applied to other polar molecules. An interesting application of the present study is the generation of electric rings currents, which depends on the sharp orientation of the molecule with respect to the excitation field. It also promotes new application for understanding and controlling molecular processes and further offers advance research in molecular science.

\section{Acknowledgements}

UA acknowledges the help from the University Grants Commission (UGC), New Delhi, India for financial support.

\section{References}

1. Kitano K, Hasegawa H and Ohshima Y 2009 Phys. Rev. Lett. 103223002

2. Herschbach D 2006 Eur. Phys. J. D 383

3. McCarthy T J, Timko M T and Herschbach D R 2006 J. Chem. Phys. 125133501

4. Lee S K, Silva R, Thamanna S, Vasyutinskii O S and Suits A G 2006 J. Chem. Phys. 125144318

5. Friedrich B and Herschbach D 2003 Phys. Today 5653

6. Loesch H J and Remschied A 1990 J. Chem. Phys. 93 4779

7. Prasad V, Sharma R, Mathur P C and Mohan M 1998 Mol. Phys. 95689

8. Singhal N, Prasad V and Mohan M 2002 Eur. Phys. J. D 21293

9. Friedrich B, Rubahn H-G and Sathyamurthy N 1992 Phys. Rev. Lett. 692487

10. Friedrich B and Herschbach D 1995 Phys. Rev. Lett. 74 4623

11. Stapelfeldt H and Seideman T 2003 Rev. Mod. Phys. 75 543

12. Arya U, Dahiya B and Prasad V 2012 Spectrochim. Acta A 95491

13. Bracker A S, Wouters E R, Suits A G and Vasyutinskii O S 1999 J. Chem. Phys. 1106749

14. Moreno P S, Ferez R G and Schmelcher P 2007 Phys. Rev. A 76053413

15. Hashmi F A and Bouchene M A 2009 Phys. Rev. A 79 025401

16. Itakura R, Hasegawa H, Kurosaki Y, Yokoyama A and Ohshima Y 2010 J. Phys. Chem. A 11411202

17. Baek D, Hasegawa H and Ohshima Y $2011 \mathrm{~J}$. Chem. Phys. 134224302

18. Hashmi F A and Bouchene M A 2010 Phys. Rev. A 82 043432

19. Bisgaard C Z, Viftrup S S and Stapelfeldt H 2006 Phys. Rev. A 73053410

20. Sugny D, Keller A, Atabek O, Daems D, Dion C M, Guerius and Jauslin H R 2004 Phys. Rev. A 69033402

21. Daems D, Guerin S, Sugny D and Jauslin H R 2005 Phys. Rev. Lett. 94153003

22. Xu S -W, Huang Y -X and Ji X -M 2011 Chin. Phys. Lett. 28043301

23. Yu J, Zhang W, Yang J and Cong S-L 2011 Chin. Phys. Lett. 28103301

24. Yun H, Kim H T, Kim C M, Nam C H and Lee J 2011 Phys. Rev. A 84065401

25. Zhang S, Lu C, Jia T, Wang Z and Sun Z 2011 J. Chem. Phys. 135034301

26. Zhang S, Lu C, Jia T, Wang Z and Sun Z 2011 Phys. Rev. A $\mathbf{8 3} 043410$

27. Lee K F, Villeneuve D M, Corkum P B and Shapiro E A 2004 Phys. Rev. Lett. 93233601 
28. Lee K F, Shapiro E A, Villeneuve D M and Corkum P B 2006 Phys. Rev. A 73033403

29. Renard M, Hertz E, Guerin S, Jauslin H R, Lavorel B and Faucher O 2005 Phys. Rev. A 72025401

30. Horn C, Wollenhaupt M, Krug M, Baumert T, Nalda R and de Banares L 2006 Phys. Rev. A 73 031401(R)

31. Anderson N A, Shiang J J and Sension R J, 1999 J. Phys. Chem. A 10310730

32. Anderson N A, Durfee C G III, Murnane M M, Kapteyn H C and Sension R J 2000 Chem. Phys. Lett. 323365

33. DeWitt M J and Levis R J, 1998 J. Chem. Phys. 1087739

34. Markevitch A N, Moore N P and Levis R J 2001 Chem. Phys. 267131

35. Kosmidis C, Tzallas P, Ledinglam K W D, McCanny T, Singhal R P, Taday P F, Langley A J 1999 J. Phys. Chem. A 1036950

36. DeWitt M J, Peters D W and Levis R J 1997 Chem. Phys. 218211

37. Smith S M, Li X, Markevitch A N, Romanov D A, Levis R J and Schlegel H B 2005 J. Phys. Chem. A 10910527

38. Miyazaki K, Kaku M, Miyaji G, Abdurrouf A and Faisal F H M2005 Phys. Rev. Lett. 95243903

39. Kanai T, Minemoto S and Sakai H 2005 Nature 435 470

40. Wetzels A et al 2001 Eur. Phys. J. D 14157
41. Tannian B E et al 2000 Phys. Rev. A 62043402

42. Baker S, Walmsley I A, Tisch J W G and Marangos J P 2011 Nature Photonics 5664

43. You D, Jones R R, Bucksbaum P H and Dykaar D R 1993 Opt. Lett. 18290

44. Jones R R, You D and Bucksbaum P H 1993 Phys. Rev. Lett. 701236

45. Bensky T J, Haeffler G and Jones R R 1997 Phys. Rev. Lett. 792018

46. Altucci C, Tisch J W G and Velotta R 2011 J. Mod. Opt. 581585

47. Lamb G L Jr 1974 Phys. Rev. A 9422

48. Haus H A 1979 Rev. Mod. Phys. 51331

49. Dion C M, Keller A and Atabek O 2001 Eur. Phys. J. D 14249

50. Barth I, Andres L S- and Seideman T 2008 J. Chem. Phys. 129164303

51. Abiague A M and Berakdar J 2003 Chem. Phys. Lett. 382475

52. Wu C, Zeng G, Jiang H, Gao Y, Xu N and Gong Q 2009 J. Phys. Chem. A 11310610

53. Wu C, Zeng G, Gao Y, Xu N, Peng L - Y, Jiang $\mathrm{H}$ and Gong Q 2009 J. Chem. Phys. 130231102

54. Zhao S, Liu P, Li Y, Li R and Xu Z 2009 Chem. Phys. Lett. 48067 